\title{
Update on the emerging role of telavancin in hospital-acquired infections
}

\author{
This article was published in the following Dove Press journal: \\ Therapeutics and Clinical Risk Management \\ 13 April 2015 \\ Number of times this article has been viewed
}

\author{
Obinna N Nnedu' \\ George A Pankey ${ }^{2}$ \\ 'Infectious Disease Department, \\ Ochsner Clinic Foundation, New \\ Orleans, LA, USA; ${ }^{2}$ Infectious Disease \\ Research, Ochsner Clinic Foundation, \\ New Orleans, LA, USA
}

\begin{abstract}
Telavancin is a lipoglycopeptide that has activity against Gram-positive aerobic and anaerobic bacteria. It has activity against methicillin-resistant Staphylococcus aureus, vancomycin-intermediate $S$. aureus and non-Van-A strains of vancomycin-resistant enterococci. It has been approved by the US Food and Drug Administration (FDA) for complicated skin and skin structure infections and hospital-acquired pneumonia. There is a need for more clinical studies to determine the role of telavancin in treating bacteremia and prosthetic device infections. In this review, we discuss the published data on the use of telavancin in treating hospital-acquired infections and provide an update on new research.
\end{abstract}

Keywords: telavancin, vancomycin, linezolid, Staphylococcus aureus

\section{Introduction}

Hospital-acquired infections cause high rates of morbidity and mortality. In addition to the human cost, the financial cost on hospitals is immense. The Centers for Disease Control and Prevention classifies hospital-acquired infections into four groups; hospital-associated pneumonia (HAP), surgical site infections (SSIs), central lineassociated bloodstream infections (CLABSIs), and catheter-associated urinary tract infections (CAUTIs). ${ }^{1}$ Many hospital-acquired infections are caused by Gram-positive bacteria. Bacteria isolated from these infections are frequently resistant to routinely used antibiotics. Effective antimicrobial treatment of incident cases is an important cornerstone for decreasing the burden of hospital-acquired infections. Telavancin is a new lipoglycopeptide that has been approved by the US Food and Drug Administration (FDA) for treating complicated skin and skin structure infections (cSSSIs) and HAP. It has a broad range of activity against aerobic and anaerobic Gram-positive bacteria. In this review, we explore the possible role of telavancin in treating HAP, SSI, CLABSI, and CAUTI.

\section{Microbiology and pharmacology}

Telavancin has antimicrobial activity against drug-resistant pathogens, including methicillin-resistant Staphylococcus aureus (MRSA), vancomycin-intermediate S. aureus (VISA), and linezolid-resistant $S$. aureus. ${ }^{2-4}$ In vitro data also suggest a potential role in treating non-Van-A strains of vancomycin-resistant Enterococcus. ${ }^{4,5}$ The Van-A gene confers resistance by altering the binding site for glycopeptides from D-Ala-D-Ala to D-Ala-D-Lac. This mutation also decreases telavancin efficacy. In vitro synergy studies have shown synergy for combination of telavancin and rifampin in treating Enterococcus faecium resistant to both linezolid and vancomycin. ${ }^{6}$ Care must be taken, however, in interpreting in vitro synergy studies as they may not correlate with clinical efficacy.
Correspondence: Obinna N Nnedu Ochsner Infectious Disease Training Program, The University of Queensland School of Medicine, Ochsner Clinical School, I5I4 Jefferson Highway, New Orleans, LA 70I2I, USA

Tel +l 5048424006

Fax + I 5048425254

Email onnedu@ochsner.org
Therapeutics and Clinical Risk Management 2015:II 605-610

605

Dovepress

http://dx.doi.org/10.2147/TCRM.S57376 (c) (i) (5) 2015 Nnedu and Pankey. This work is published by Dove Medical Press Limited, and licensed under Creative Commons Attribution - Non Commercial (unported, v3.0) License. The full terms of the License are available at http://creativecommons.org/licenses//by-nc/3.0/. Non-commercial uses of the work are permitted without any further peres the license ane administered by Dove Medical Press Limited. Information on how to request permission may be found at: http://www.dovepress.com/permissions.php 
A postmarketing study of 15,480 Gram-positive bacterial isolates from 89 countries obtained in 2010 showed that telavancin still maintained potent activity against these bacterial isolates. ${ }^{7}$ In the study, telavancin had low minimum inhibitory concentration (MIC) for methicillin-sensitive S. aureus (MSSA) (0.03-0.5), MRSA $(<0.015-0.5)$, and coagulase-negative staphylococci $(<0.015-1){ }^{7}$

The FDA approved a revised broth microdilution method for telavancin in $2014{ }^{8}$ This new method is used for other lipoglycopeptides and provides more accurate MICs. MICs for Gram-positive organisms utilizing this method have been found to be lower, suggesting greater efficacy than previously thought. Utilizing this new broth microdilution method and new FDA breakpoints, a study of 10,920 nonduplicate isolates collected between 2011 and 2012 confirmed high-level activity against Staphylococcus, Streptococcus, and vancomycin-susceptible Enterococcus. ${ }^{9}$ The study also confirmed that telavancin has decreased activity against Van-A strains of vancomycin-resistant enterococci.

Telavancin has also shown activity against a number of anaerobes, including Actinomyces, Clostridium species including C. difficile, Eubacterium, Lactobacillus, Propionibacterium, Peptostreptococcus, and Corynebacterium. ${ }^{10}$ However, there is a paucity of clinical studies looking at the role of telavancin to treat infections caused by anaerobic bacteria.

Telavancin has a hydrophobic side chain on the vancosamine sugar and a hydrophilic group in the $4^{\prime}$ position of amino acid $7 .{ }^{11}$ These properties allow telavancin to have a twofold mechanism of action. Firstly, it binds to the D-Ala D-Ala moiety on peptidoglycan precursors, disrupting the late stages of cell wall synthesis. ${ }^{11}$ Secondly, telavancin disrupts cell membrane potential leading to leakage of cytoplasmic material, and cell death. ${ }^{11}$ The dual mechanism of action allows for telavancin to have fourfold enhanced bactericidal activity as compared to vancomycin when treating MRSA infection in experimental mouse models. ${ }^{12}$

Telavancin exhibits concentration-dependent bactericidal activity. ${ }^{12}$ The half-life of telavancin is 7-9 hours with a postantibiotic effect of 4-6 hours. ${ }^{13,14}$ These properties allow telavancin to be conveniently dosed once a day. The most important pharmacokinetic marker influencing efficacy is the ratio between area under the serum-concentration-vs-time curve vs MIC or area under the curve (AUC)/MIC ratio. ${ }^{12}$ Telavancin has been shown to be efficacious with AUC/MIC ratios $>219 .{ }^{12}$ Pharmacokinetic studies have shown that doses of $10 \mathrm{mg} / \mathrm{kg}$ in individuals with normal kidney function achieve adequate AUC/MIC ratios. ${ }^{14}$ About $90 \%$ of administered telavancin is bound by albumin. ${ }^{13}$ It is primarily excreted unchanged by the kidney. ${ }^{14}$ Hence for those with impairment in their kidney function, the dose has to be adjusted.

\section{Efficacy Hospital-associated pneumonia}

HAP is defined as a pneumonia that starts 48 hours or more after hospitalization. HAP may be divided into ventilator and nonventilator lung infections. The Assessment of Telavancin for Treatment of Hospital-Acquired Pneumonia (ATTAIN) studies I and II were Phase III trials that looked at the efficacy of telavancin for treating HAP. ${ }^{15}$ Both trials were designed as noninferiority trials comparing telavancin to vancomycin. Data pooled from both studies $(n=654)$ show similar cure rates between telavancin $(82 \%)$ and vancomycin (81\%) among clinically evaluable patients.

When study patients were restricted to those with S. aureus as the sole pathogen $(\mathrm{n}=298)$, cure rates were $86 \%$ for the telavancin arm and $75 \%$ for the vancomycin arm. ${ }^{15}$ In a subanalysis of only nonventilator-associated HAP $(n=519)$, cure rates were similar in the telavancin arm and in the vancomycin arm (83\% vs $84 \%) .{ }^{16}$ When the analysis was further restricted to those with MRSA-positive initial cultures $(n=223)$, there was again no statistically significant difference between cure rates in the telavancin arm (74.8\%) vs the vancomycin arm (79.3\%). ${ }^{16}$ Based on these data, the FDA has approved telavancin for use in treating HAP.

Another subgroup analysis was performed within the ATTAIN trials to compare efficacy of telavancin vs vancomycin for treating HAP associated with bacteremia. ${ }^{17}$ Bacteremic HAP was defined as patients with blood cultures positive for the same causative pathogen as the HAP. Fiftynine participants had Gram-positive bacteremia with HAP. Cure rates were $41 \%$ in the telavancin arm and $40 \%$ in the vancomycin arm. In patients with MRSA bacteremic HAP $(n=33)$, cure rates were $42 \%$ for the telavancin arm and $33 \%$ for the vancomycin arm. However, the study numbers were small and the results must be interpreted with caution.

Safety analysis of the ATTAIN trials was reported. Rates of adverse events were similar across both groups. However, severe adverse effects were higher among the telavancin arm as compared to the vancomycin arm (21\% vs $16 \%)$. The rates of treatment emergent adverse effects leading to study discontinuation were also higher in the telavancin arm as compared to the vancomycin arm (8\% vs 5\%). Clinically significant renal function decline was also seen at a higher rate among the telavancin arm as compared to the vancomycin arm (16\% vs $10 \%)$. On the basis of these data, 
patients receiving telavancin should be monitored closely with particular attention to renal function.

\section{Complicated skin and skin structure infections}

To understand the potential role of telavancin in treating SSIs, we reviewed the data on its use in treating cSSSIs. The telavancin vs standard therapy for cSSSIs caused by Gram-positive organisms (FAST) trials were two clinical trials comparing telavancin to standard care (vancomycin or anti-staphylococcal penicillin). ${ }^{18}$ In the first trial, the clinically evaluable population was 141 . Cure was achieved in $79 \%$ of the telavancin arm vs $80 \%$ of the standard care arm. Among those study participants who were culture-positive for MRSA at baseline ( $n=48), 82 \%$ of patients in the telavancin arm achieved cure vs $69 \%$ in the standard care arm. Adverse events that could have possibly been related to therapy were seen in $32 \%$ in the telavancin arm as compared to $29 \%$ in the standard care arm. Vomiting, paresthesias, and dyspnea were seen at slightly higher rates in the telavancin arm. Severe adverse events were seen in fewer patients in the telavancin arm (3\%) vs the standard care arm $(7 \%)$.

The second trial by the FAST study group produced similar results as the first. ${ }^{19}$ While patients with creatinine clearance less than $50 \mathrm{~mL} / \mathrm{min}$ were excluded from the first trial, the second trial included these individuals. Telavancin was renally dosed in this trial. The total number of clinically evaluable participants followed up to the test of cure visit was 154 . Ninety-six percent of those in the telavancin arm achieved cure, which was comparable to $94 \%$ in the standard care arm (vancomycin or anti-staphylococcal penicillin). Rates of adverse events in both study arms were similar, 56\% for telavancin and $57 \%$ for standard care. Rates of severe adverse events were also similar amongst both groups; $6 \%$ for the telavancin group and $4 \%$ for the standard care group.

The Assessment of Telavancin in Skin and Skin Structure Infections (ATLAS I and ATLAS II) trials were Phase III trials that also looked at the role of telavancin in treating cSSSIs. ${ }^{20}$ In these trials, telavancin at a dose of $10 \mathrm{mg} / \mathrm{kg}$ every 24 hours was compared to vancomycin $1 \mathrm{~g}$ every 12 hours. Pooling both studies, 579 participants had MRSA infections. Within this group, $91 \%$ of participants in the telavancin arm achieved cure compared to $84 \%$ in the vancomycin arm. ${ }^{20}$ The ATLAS trials formed the basis for FDA approval of telavancin for cSSSIs. A subanalysis of patients in the ATLAS trials focused on SSIs was also performed. ${ }^{21}$ Telavancin was compared to vancomycin. There were no significant differences between groups due to the small sample sizes. An analysis combining the ATLAS clinical trials showed that telavancin has a decreased efficacy among patients with moderate to severe renal function. ${ }^{22}$ Among those with creatinine clearance below $50 \mathrm{~mL} / \mathrm{min}$, cure rates were $67.4 \%$ in the telavancin arm as compared to $82.7 \%$ in the vancomycin arm. The reason for this finding is unclear. However, a study utilizing serum from renal disease patients following a dose of telavancin showed that in vitro activity of telavancin was maintained against a strain of $S$. aureus. ${ }^{23}$

In the ATLAS trials, adverse events were reported for $79 \%$ of the telavancin arm vs $72 \%$ for the vancomycin arm. The most common side effects in the telavancin arm were taste disturbance, nausea/vomiting, and foamy urine. Serious adverse events were reported in $7 \%$ of the telavancin arm as compared to $5 \%$ of the vancomycin arm. The serious adverse events in the telavancin arm were primarily renal, respiratory, and cardiac events. ${ }^{24}$

An important subset of soft tissue infections are complicated by prosthetic material. There is a lack of clinical trials evaluating the role of telavancin in treating staphylococcal infections producing biofilm. Biofilm production is known to complicate treatment of prosthetic device infections. The role of telavancin in treating infections complicated by prosthetic devices has been evaluated in vitro. ${ }^{25}$ Biofilm producing strains of staphylococci and enterococci were tested against telavancin and vancomycin. Comparing concentrations of $0-16 \mu \mathrm{g} / \mathrm{mL}$, telavancin was more active against bacteria embedded in biofilms and inhibited new biofilm formation. At least two other in vitro studies have also shown that telavancin has greater activity against biofilm producing strains of Staphylococcus species. ${ }^{26,27}$ There have also been a few case reports showing successful treatment of infections complicated by prosthetic material..$^{28}$ There is a need for more clinical studies addressing this question as conclusions cannot be made from in vitro data and case reports.

\section{Central line-associated bloodstream infections}

CLABSI are frequently caused by Gram-positive bacteria. Telavancin, with its enhanced bactericidal activity against Gram-positive bacteria, could potentially play a role in treating these infections. The ASSURE (telavancin for treatment of uncomplicated bacteremia) trial was a Phase II clinical trial that compared telavancin and standard care (vancomycin or anti-staphylococcal penicillin) for the treatment of uncomplicated S. aureus bacteremia. ${ }^{29}$ Intravenous catheters were the most common cause of $S$. aureus bacteremia in this study. Fifty percent of patients had infections 
with MRSA. The clinically evaluable group within this study consisted of those who received study drugs for at least 12 days. There were eight patients in the telavancin arm and nine patients in the standard care arm among the clinically evaluable study participants. Cure rates were comparable across both groups, suggesting a potential role for telavancin in treating these infections. Given the small numbers in this Phase II trial, additional studies are needed before making any definitive conclusions. The small sample size of the ASSURE trial also means that safety data from this study may not be accurate. In this study, $90 \%$ of the people in the telavancin arm had at least one adverse event compared to $72 \%$ in the standard care arm. The most common adverse events were fever, headache, and rash.

There have been case reports showing successful use of telavancin in the treatment of MRSA bacteremia cases. One case report outlined the successful use of telavancin to treat a patient with persistent MRSA bacteremia in the setting of endocarditis. ${ }^{30}$ In this case, the patient had remained bacteremic for 10 days despite therapeutic vancomycin levels. After switching to telavancin $10 \mathrm{mg} / \mathrm{kg}$, blood cultures became negative. Follow-up cultures 6 months after completion of therapy were also negative. ${ }^{30}$ Another case report outlined a successful treatment of VISA pacemaker lead infective endocarditis with combination of lead removal and telavancin. ${ }^{31}$ Joson et $\mathrm{al}^{32}$ have also published a case of successful treatment of mitral valve endocarditis with telavancin.

A study on the use of telavancin in treating bloodstream infections was presented at the 2014 Infectious Diseases Society of America meeting. ${ }^{33}$ In this study, 78 cancer patients with Gram-positive bloodstream infections received either vancomycin or telavancin. The bacterial isolates found were $S$. aureus (51\%), followed by alpha-hemolytic streptococci (23\%), Enterococcus (15\%), coagulase-negative Staphylococcus ( $8 \%$ ), and beta-hemolytic streptococci (3\%). Fifty-one percent of participants were neutropenic at onset of therapy. There was a trend toward better clinical response in the telavancin arm as compared to the vancomycin arm (83.3\% vs $63.2 \%$; $P=0.051)$, but this did not achieve statistical significance. ${ }^{33}$ There have been review papers outlining existing data on the use of telavancin to treat bloodstream infections. ${ }^{34,35}$ Additional studies are needed to fully determine the role telavancin could play in Gram-positive endocarditis.

\section{Catheter-associated urinary tract infections}

Telavancin is primarily excreted by the kidneys. However, to our knowledge, there have not been any studies evaluating the role of telavancin in treating urinary tract infections. There is a need to look at the utility of this antibiotic for treating resistant Gram-positive causes of lower and upper urinary tract infections such as vancomycin-resistant E. faecium.

\section{Safety and tolerability}

A pooled analysis of five randomized controlled trials was performed which compared rates of mortality between telavancin and vancomycin groups. ${ }^{36}$ Data for this analysis were available for 3,565 patients. The rates of mortality were similar between telavancin and vancomycin groups; $8.9 \%$ vs $8.3 \%$, $\mathrm{OR}=1.08(95 \% \mathrm{CI}, 0.84-1.38) .{ }^{36}$ However, among those with kidney dysfunction, data from a clinical trial of patients with HAP showed lower 28-day survival rates with telavancin as compared to vancomycin. ${ }^{37}$ Among patients with moderate to severe kidney dysfunction (creatinine clearance $<50 \mathrm{~mL} / \mathrm{min}$ ), 28-day survival rates for telavancin (59\%) were lower than vancomycin (70\%). ${ }^{37}$ When analysis was further restricted to patients with very severe kidney disease (creatinine clearance $<30 \mathrm{~mL} / \mathrm{min}$ ), 28-day survival was again lower in the telavancin arm (47\%) vs the vancomycin arm $(62 \%) .{ }^{37}$

Telavancin has also been associated with higher rates of acute kidney injury. ${ }^{36}$ Pooled analysis of six randomized controlled trials $(3,312$ total patients) showed a $10 \%$ rate of kidney injury in the telavancin group compared to the vancomycin group at $5 \%, \mathrm{OR}=2.22(95 \% \mathrm{CI}, 1.38-3.57){ }^{36}$ To our knowledge, telavancin did not cause end-stage renal disease in any of the study participants. Patients with preexisting kidney disease need to be monitored very closely if they are placed on telavancin. Besides acute kidney injury, other reported adverse events among those receiving telavancin included taste disturbance $(31.6 \%)$, nausea $(17.1 \%)$, headache (13.2\%), constipation (9.3\%), insomnia (7.7\%), anemia (6.3\%), and corrected QT interval prolongation $(3.8 \%) .{ }^{36}$ Telavancin has not been studied in pregnant women. However, in animal models, telavancin was associated with birth defects. Hence it should not be used in pregnant women.

\section{Cost}

An important factor that influences the use of an antibiotic is cost. Pharmacoeconomic analysis which compared the cost of telavancin against vancomycin for treatment of cSSSIs was performed. ${ }^{38}$ Data from the ATLAS Phase III clinical trial was utilized. This analysis was restricted to study participants who had been hospitalized. Variables in this analysis included infection-related length of hospital stay, infection-related cost, cost of vancomycin monitoring, and antibiotic acquisition prices. The sensitivity of their analysis was tested by incrementally increasing the cost per dose of telavancin up to 15 times the cost per dose of 
Table I Cure rates of telavancin for treating infections

\begin{tabular}{|c|c|c|}
\hline Clinical syndrome (sample size) & Telavancin (cure rate \%) & $\begin{array}{l}\text { Vancomycin or anti-staphylococcal } \\
\text { penicillin (cure rate } \% \text { ) }\end{array}$ \\
\hline $\operatorname{HAP}^{a}(n=654)$ & 82 & 81 \\
\hline $\mathrm{cSSSI}^{\mathrm{b}}(\mathrm{n}=|4|)$ & 79 & 80 \\
\hline cSSSI secondary to $\operatorname{MRSA}^{c}(n=579)$ & 91 & 84 \\
\hline Bloodstream infection ${ }^{d}(n=17)$ & 88 & 89 \\
\hline Urinary tract infections & No data & No data \\
\hline
\end{tabular}

Notes: a ATTAIN trials ${ }^{15}$; ${ }^{\mathrm{F}} \mathrm{AST}$ trials ${ }^{18}$; ${ }^{\mathrm{A}} \mathrm{TLAS}$ trials ${ }^{20}$; ${ }^{\mathrm{d}}$ ASSURE trials. ${ }^{29}$

Abbreviations: HAP, hospital-associated pneumonia; cSSSI, complicated skin and skin structure infection; MRSA, methicillin-resistant Staphylococcus aureus.

vancomycin. The analysis found no significant difference in infection-related length of stay between telavancin and vancomycin.

There was also no significant difference in infectionrelated cost.

\section{Conclusion}

A summary of the cure rates of telavancin for treating infections is provided in Table 1. Data from clinical trials show that telavancin may be of particular use in the treatment of cSSSIs and HAP. There is a need for more clinical data on its use in treating bloodstream and urinary tract infections. In choosing an antibiotic for patients, clinicians would need to factor in drug efficacy, side effects, convenience, and cost. Telavancin has enhanced bactericidal activity against a range of drug-resistant Gram-positive organisms. It is also dosed once a day. However, acute kidney injury was noted in higher rates among those receiving telavancin as compared to vancomycin. Telavancin does not require therapeutic drug level monitoring as vancomycin does and may have a comparable overall cost to vancomycin. ${ }^{38}$ Additional clinical studies are needed to determine the role of telavancin in the treatment of CLABSI and CAUTI.

\section{Disclosure}

The authors report no conflicts of interest in this work.

\section{References}

1. Types of Healthcare-associated Infections [page on the Internet]. Atlanta: Centers for Disease Control and Prevention; c2014 [updated March 26, 2014; cited December 1, 2014]. Available from: http://www.cdc.gov/ HAI/infectionTypes.html. Accessed February 9, 2015.

2. Leuthner KD, Cheung CM, Rybak MJ. Comparative activity of the new lipoglycopeptide telavancin in the presence and absence of serum against 50 glycopeptide nonsusceptible staphylococci and three vancomycin-resistant Staphylococcus aureus. J Antimicrob Chemother. 2006;58(2):338-343.

3. Barcia-Macay M, Lemaire S, Mingeot-Leclercq MP, Tulkens PM, Van Bambeke F. Evaluation of the extracellular and intracellular activities (human THP-1 macrophages) of telavancin versus vancomycin against methicillin-susceptible, methicillin-resistant, vancomycinintermediate, and vancomycin-resistant Staphylococcus aureus. J Antimicrob Chemother. 2006;58(6):1177-1184.
4. Krause KM, Renelli M, Difuntorum S, Wu TX, Debabov DV, Benton BM In vitro activity of telavancin against resistant gram-positive bacteria. Antimicrob Agents Chemother. 2008;52(7):2647-2652.

5. Jansen WT, Verel A, Verhoef J, Milatovic D. In vitro activity of telavancin against grampositive clinical isolates recently obtained in Europe. Antimicrob Agents Chemother. 2007;51(9):3420-3424.

6. Pankey GA, Ashcraft DS. In vitro synergy of telavancin and rifampin against Enterococcus faecium resistant to both linezolid and vancomycin. Ochsner J. 2013;13(1):61-65.

7. Mendes RE, Sader HS, Farrell DJ, Jones RN. Worldwide appraisal and update (2010) of telavancin activity tested against a collection of Gram-positive clinical pathogens from five continents. Antimicrob Agents Chemother. 2012;56(7):3999-4004.

8. Farrell DJ, Mendes RE, Rhomberg PR, Jones RN. Revised reference broth microdilution method for testing telavancin: effect on MIC results and correlation with other testing methodologies. Antimicrob Agents Chemother. 2014;58(9):5547-5551.

9. Mendes RE, Farrell DJ, Sader HS, Flamm RK, Jones RN. Baseline activity of telavancin against Gram-positive clinical isolates responsible for documented infections in US hospitals (2011-2012) as determined by the revised susceptibility testing method. Antimicrob Agents Chemother. 2015;59(1):702-706.

10. Goldstein EJ, Citron DM, Merriam CV, Warren YA, Tyrrell KL, Fernandez HT. In vitro activities of the new semisynthetic glycopeptide telavancin (TD-6424), vancomycin, daptomycin, linezolid, and four comparator agents against anaerobic gram-positive species and Corynebacterium spp. Antimicrob Agents Chemother. 2004;48(6): 2149-2152.

11. Higgins DL, Chang R, Debabov DV, et al. Telavancin, a multifunctional lipoglycopeptide, disrupts both cell wall synthesis and cell membrane integrity in methicillin-resistant Staphylococcus aureus. Antimicrob Agents Chemother. 2005;49(3):1127-1134.

12. Hegde SS, Reyes N, Wiens T, et al. Pharmacodynamics of telavancin (TD-6424), a novel bactericidal agent, against gram-positive bacteria. Antimicrob Agents Chemother. 2004;48(8):3043-3050.

13. Shaw JP, Seroogy J, Kaninga K, Higgins DL, Kitt M, Barriere S. Pharmacokinetics, serum inhibitory and bactericidal activity, and safety of telavancin in healthy subjects. Antimicrob Agents Chemother. 2005;49(1):195-201.

14. Plotkin P, Patel K, Uminski A, Marzella N. Telavancin (vibativ), a new option for the treatment of gram-positive infections. P T. 2011;36(3): $127-138$.

15. Rubinstein E, Lalani T, Corey GR, et al. Telavancin vs vancomycin for hospital-acquired pneumonia due to gram-positive pathogens. Clin Infect Dis. 2011;52(1):31-40.

16. Rubinstein E, Stryjewski ME, Barrierre SL. Clinical utility of telavancin for treatment of hospital-acquired pneumonia: focus on non-ventilatorassociated pneumonia. Infect Drug Resist. 2014;7:129-135.

17. Stryjewski ME, Barrierre SL, Rubinstein E, et al. Telavancin vs vancomycin for bacteraemic hospitalized-acquired pneumonia. Int $J$ Antimicrob Agents. 2013;42(4):367-369. 
18. Stryjewski ME, O'Riordan WD, Lau WK, et al. Telavancin versus standard therapy for treatment of complicated skin and soft-tissue infections due to gram-positive bacteria. Clin Infect Dis. 2005;40(11): 1601-1607.

19. Stryjewski ME, Chu VH, O'Riordan WD, et al. Telavancin versus standard therapy for treatment of complicated skin and skin structure infections caused by gram-positive bacteria: FAST 2 study. Antimicrob Agents Chemother. 2006;50(3):862-867.

20. Stryjewski ME, Graham DR, Wilson SE, et al. Telavancin versus vancomycin for the treatment of complicated skin and skin-structure infections caused by gram-positive organisms. Clin Infect Dis. 2008;46(11):1683-1693.

21. Wilson SE, O'Riordan W, Hopkins A, Friedland HD, Barriere SL, Kitt MM; ATLAS investigators. Telavancin versus vancomycin for the treatment of complicated skin and skin-structure infections associated with surgical procedures. Am J Surg. 2009;197(6):791-796.

22. VIBATIV [package insert]. South San Francisco, CA: Theravance, Inc.; 2013. Available from: www.vibativ.com. Accessed August 7, 2014.

23. Barriere SL, Farrell DJ, Rhomberg PR, Jones RN. Serum inhibitory and bactericidal activity of telavancin in non-infected subjects with severe renal impairment or end-stage renal disease. Diagn Microbiol Infect Dis. 2014;80(4):327-329.

24. Barriere SL. ATLAS trials: efficacy and safety of telavancin compared with vancomycin for the treatment of skin infections. Future Microbiol. 2010;5(12):1765-1773.

25. LaPlante KL, Mermel LA. In vitro activities of telavancin and vancomycin against biofilmproducing Staphylococcus aureus, S. epidermidis, and Enterococcus faecalis strains. Antimicrob Agents Chemother. 2009;53(7):3166-3169.

26. Smith K, Gemmell CG, Lang S. Telavancin shows superior activity to vancomycin with multidrug-resistant Staphylococcus aureus in a range of in vitro biofilm models. Eur J Clin Microbiol Infect Dis. 2013;32(10): $1327-1332$.

27. Gander S, Kinnaird A, Finch R. Telavancin: in vitro activity against staphylococci in a biofilm model. J Antimicrob Chemother. 2005;56(2): 337-343.
28. Kaushal R, Hassoun A. Successful treatment of methicillin-resistant Staphylococcus epidermidis prosthetic joint infection with telavancin. J Antimicrob Chemother. 2012;67(8):2052-2053.

29. Stryjewski ME, Lentnek A, O'Riordan W, et al. A randomized Phase 2 trial of telavancin versus standard therapy in patients with uncomplicated Staphylococcus aureus bacteremia: the ASSURE study. $B M C$ Infect Dis. 2014;14:289.

30. Nace H, Lorber B. Successful treatment of methicillin-resistant Staphylococcus aureus endocarditis with telavancin. J Antimicrob Chemother. 2010;65(6):1315-1316.

31. Marcos LA, Camins BC. Successful treatment of vancomycin-intermediate Staphylococcus aureus pacemaker lead infective endocarditis with telavancin. Antimicrob Agents Chemother. 2010;54(12):5376-5378.

32. Joson J, Grover C, Downer C, Pujar T, Heidari A. Successful treatment of methicillinresistant Staphylococcus aureus mitral valve endocarditis with sequential linezolid and telavancin monotherapy following daptomycin failure. J Antimicrob Chemother. 2011;66(9):2186-2188.

33. Chaftari A, Hachem R, Jordan M, et al. Evaluation of telavancin in the treatment of Grampositive bloodstream infections in cancer patients. Poster presented at: IDWeek; October 8-12, 2014; Philadelphia, PA.

34. Corey GR, Rubinstein E, Stryjewski ME, Bassetti M, Barriere SL. Potential role for telavancin in bacteremic infections due to Grampositive pathogens: focus on Staphylococcus aureus. Clin Infect Dis. Epub December 3, 2014. pii: ciu971.

35. Kenyan Y, Rubinstein E. Telavancin in the treatment of invasive Grampositive infections. Clin Audit. 2012;4:25-29.

36. Polyzos KA, Mavros MN, Vardakas KZ, Makris MC, Rafailidis PI, Falagas ME. Efficacy and safety of telavancin in clinical trials: a systematic review and meta-analysis. PLoS One. 2012;7(8):e41870.

37. Corey GR, Kollef MH, Shorr AF, et al. Telavancin for hospital-acquired pneumonia: clinical response and 28-day survival. Antimicrob Agents Chemother. 2014;58(4):2030-2037.

38. Laohavaleeson S, Barriere SL, Nicolau DP, Kuti JL. Cost-effectiveness of telavancin versus vancomycin for treatment of complicated skin and skin structure infections. Pharmacotherapy. 2008;28(12):1471-1482.
Therapeutics and Clinical Risk Management

\section{Publish your work in this journal}

Therapeutics and Clinical Risk Management is an international, peerreviewed journal of clinical therapeutics and risk management, focusing on concise rapid reporting of clinical studies in all therapeutic areas, outcomes, safety, and programs for the effective, safe, and sustained use of medicines. This journal is indexed on PubMed Central, CAS,

\section{Dovepress}

EMBase, Scopus and the Elsevier Bibliographic databases. The manuscript management system is completely online and includes a very quick and fair peer-review system, which is all easy to use. Visit http://www.dovepress.com/testimonials.php to read real quotes from published authors. 